\title{
Algerian Judo Competition Modality and its Impacts on Upper and Lower Limbs Strength Perseverance and Limitations
}

\author{
Belkadi Adel*, Charara Alia and Zerf Mohammed \\ Institute of Physical Education and Sport-University of Mostaganem, Algeria \\ *Corresponding author: Belkadi Adel, Institute of Physical Education and Sport -University of Mostaganem, Algeria
}

\begin{abstract}
Objective: This study was designed to analyze the impact of Algerian judo competition modality on vertical jump performance, grip strength test and perceived effort responses.

Method: For the proposed 34 male judoka athletes participated in the present study. All their performance was verified based on the Algeria modality competition that consisted form 4 min fight separated by 15 min of rest. Judo-Ji grip strength tests (JGST), countermovement jump (CMJ) and rate of perceived exertion (RPE) were measured before and after each competition, while RPE of specific areas was assessed after three competitions. ANOVA for repeated measures was used to compare strength parameters after each contest with the level of significance set at $5 \%$.

Result: our results showed a significant decrease of jump height $(p=0.001)$ and vertical impulse in the CMJ $(p=0.00)$, as well as a reduction of the number of reps in the JGST $(p<0.00)$. In the opposite of significant increase of RPE throughout the competition $(\mathrm{p}<0.00)$, not RPE between the upper and lower body $(\mathrm{p}=0.00)$.
\end{abstract}

Conclusion: We conclude that Algerian modality judo competition generated a decrease of performance in both upper and lower limbs and provoked a progressive increase in the effort perception over the fighter.

Keywords: Competition; Strength Parameters; Perceptual Responses Judo Athletes

\section{Introduction}

Judo is a full-contact martial art originating from ancient Japan and has developed into an Olympic sporting event [1]. It's also intermittent and comprised of high-intensity periods of effort and short periods of rest [2], in the competitive period high-intensity combat could be prioritized, considering official competition duration, and intervals between fights similar to those established in competitions or long enough to provide full recovery for athletes [3]. To be successful in international competitions, judo athletes must achieve an excellent level of physical fitness and physical condition during training [4] noted that athletes having little chance of properly applying takedowns or submission techniques in the most judo competition championships.

The effort perception is another variable that also provides important information for comprehending the impairment of performance due to fatigue [5]. The rating of perceived exertion (RPE) is a recognized marker of intensity and of homeostatic disturbance during exercise [6] and It is monitored during exercise tests to complement other measures of intensity [7] The RPE in specific muscles showed 21 reports in ten areas with an intensity close to 15 (hard) [3]. It'simperativetocontrolseveralfactorsbyimprovingexternalvalidity during the competition. As well as enhance the accuracy of fatigue analysis, such as fights with different opponents, within the same weight category and training level, number of fights in the competition, competition time, intervals between each competition and the minimum time to assess the physical responses [8]. For this reason, we tested our competitive judoka players to estimate the impact of the Algerian judo competition mode on upper and lower limbs strength perseverance and limits. Where our objectives consist to controllers of those aforementioned factors. The gathered information that may allow our trainers a better 
understanding of the effort of an internal load during successive competition and, consequently, improve judo-specific strength training periodization. Driven by the study of [9] in the choice of tests Squat Jump, and Countermovement Jump between judo-and non-judo athletes [10]. As well as to test the main hypothesis in which we suppose that mode of Algerian Competition induced a decrease of strength/power performance in both upper and lower limbs and provoked a progressive increase in the effort perception over the system of Algerian Competition.

\section{Methods}

Participants: Thirty-four male judo athletes (age $=21.8 \pm$ 2.9 years; body mass $=69.2 \pm 3.1 \mathrm{~kg}$; height $=1.75 \pm 0.05 \mathrm{~m}$; body fat $=10.5 \pm 2.5 \%$ ) volunteered to participate in this study. The length of time previously practising judo was $6.7 \pm 4.7$ years. 17 athletes were 1 dan black belts and seventeen were brown belts. All athletes had already participated in national and international judo competitions. They were regularly training 4-5 times a week during the evaluation period. Participants were selected based on the following criteria: they had no reported musculoskeletal disorder or injury that influenced their maximal physical performance, and they were required to have been training regularly for at least 5 years. They were in the competition preparatory phase and therefore, not in a period of rapid weight loss. All participants were informed about the study procedures. The Institute of physical education and sports of Mostaganem Research Ethics Committee approved this study (protocol number: 0806/IEPS-20 September 2017).

\section{Experimental Protocol}

Judoka athletes participated in a competition consisting of five 4 min competition separated by $10 \mathrm{~min}$ of passive rest (Figure 1). Before the competition their body mass was measured on a scale with $0.1 \mathrm{~kg}$ accuracy, height with a stadiometer with $0.1 \mathrm{~cm}$ accuracy, and four skinfolds (triceps, subscapular, super-iliac and abdominal) with an adipometer of $0.1 \mathrm{~cm}$ of accuracy.

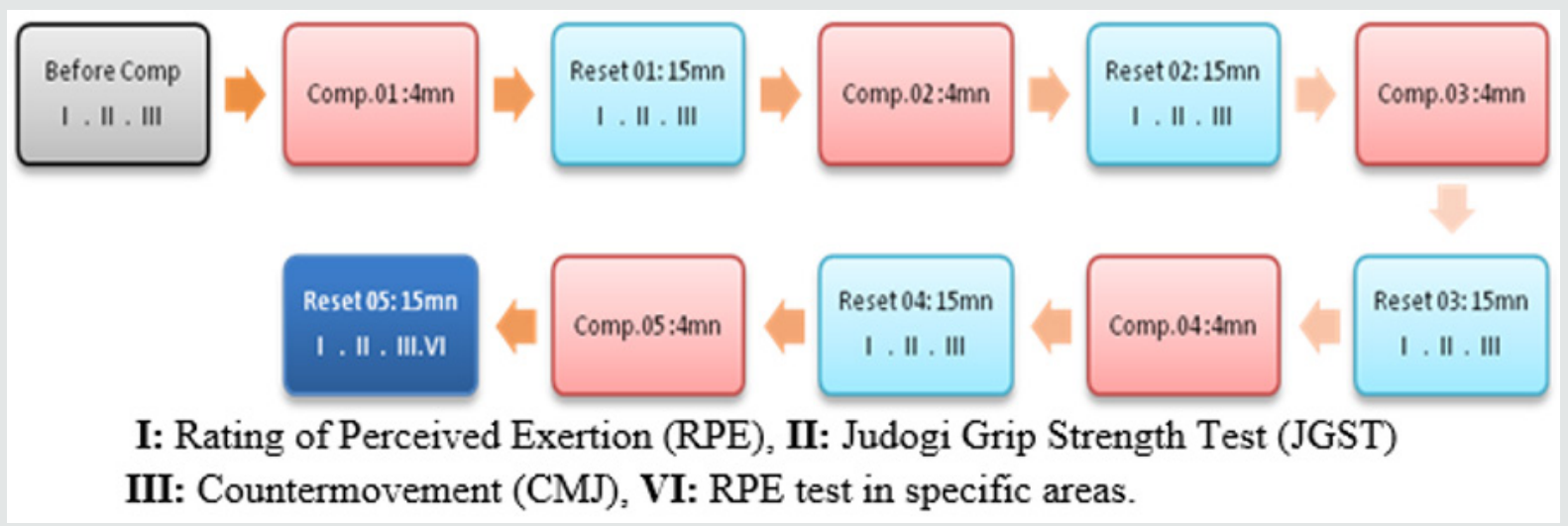

Figure 1: Experimental design of Judo Competition.

The Body Composition and Somatotype were obtained by the equation proposed by [11]. The somatic profile of body build for athletes in this sport seems to be optimal for achieving high results [12], To test the effects of the competition on muscle strength in the upper and lower limbs, the Judo-Ji grip strength test (JGST) and countermovement jump (CMJ) were measured before the first competition and after each competition. These tests were performed in a randomized order as fast as possible and lasted from 09 to $12 \mathrm{~min}$ after each competition (first, second, third, semi-final and final). The time between the assessments was determined during a pilot study since it was the minimum time to perform all measures. The RPE was assessed before the first competition and immediately after each competition [13] noted that it can be used in such diverse fashions as to predict exercise capacity, assess changes in training status, and explain changes in pace and pacing strategy. In addition to using RPE to self-regulate exercise, a new application of the intensity; RPE relationship is to clamp RPE at various levels to produce self-paced bouts of exercise [14], which can be used to assess maximal functional capacity. The perception of effort in specific areas was assessed only after the last competition. The competition was performed in the morning (10:00-16:30 am) within a room temperature ranging from 22 to $24{ }^{\circ} \mathrm{C}$. Participants were instructed to maintain normal diets and prior to the assessments and training session. In addition, it was recommended to avoid any physical training $72 \mathrm{~h}$ before data collection to before event possible muscle damage. [15] have noted no changes were observed in the physiological, rating of perceived exertion, or technical actions during 3 comp competition.

\section{Judo competition}

The Judo athletes performed five 4 min competition separated by $15 \mathrm{~min}$ of passive rest. This length of time for combat and intervals may be considered an intermediate level. According to the IJF Judo Refereeing Rules (" Documents / IJF.org », s. d.), the time competition is $4 \mathrm{~min}$ to unlimited time (golden score), respectively, with the interval between each competition at least three times of the competition. All competitions were performed following the official IJF rules. The athletes were informed that they must complete all five competition. In the event of submission (the event that normally determines the end of combat), the fight was restarted to guarantee that all athletes competed for the same duration of time in all competitions. During the break periods, the athletes were encouraged to drink water to maintain hydration levels, but they did not ingest any food during the competition. The participants were divided into pairs in the same weight category and with a difference of body mass of no more than $10 \%$ between 
them. This protocol real judo combat activity (temporal structure and category) as described in the correlating literature [16]. In addition, the athletes fought with a different opponent for each of the five competitions. This procedure ensured a more accurate of real competition.

\section{Countermovement jump evaluation}

Before combat, the participants performed the familiarization/ warm-up exercises, consisting of 1 min of hopping on a trampoline, three series of ten hops on the Tatami, and 8-10 sub-maximal CMJs. After a 10 min resting period, they performed five maximal CMJs on a piezoelectric force platform (Model 9290AD, Quattro Jump) a frequency of $500 \mathrm{~Hz}$. This test was repeated after competition from one to five times. During the CMJs, the participants were asked to sustain the trunk as vertical as possible, while the hands were placed on the hips. Additionally, they were requested to flex their knees in the transition between the eccentric-concentric phases. Ground reaction force (GRF) data were filtered by a fourth-order low-pass Butterworth filter at $20 \mathrm{~Hz}$, determined from power spectral analysis $[16,17]$ shows that the net vertical impulse was determined during the propulsive phase of the $\mathrm{CMJ}$ from the point when displacement values reached a maximum depth during the bottom of the coupling phase until the force-time curve returned to zero (Figure 2). Net impulse was calculated by removing the vertical impulse exerted through the acceleration due to gravity. Then, the values were divided by the body mass of each participant to determine the relative net vertical impulse. The power output was calculated by multiplying the GRF by velocity in the ascent phase of the jump (from when the Centre of mass velocity becomes positive until take-off) (Figure 2) The mean value of the curve was used for analysis. An algorithm implemented in MATLAB ${ }^{\text {TM }}$ software (MathWorks, Natick, MA, USA) was used in the analysis (Figure 2). Mean values of jump height, power output and relative net vertical impulse in each evaluation (Before-Competition, postcompetition one, two, three semi-final and final) were considered for the posterior analysis. The reliability of CMJ variables was calculated by the three baseline trials. We found a contrast intrasubjects correlation coefficient (ICC) ranging from 0.97 to 0.99 for all variables.

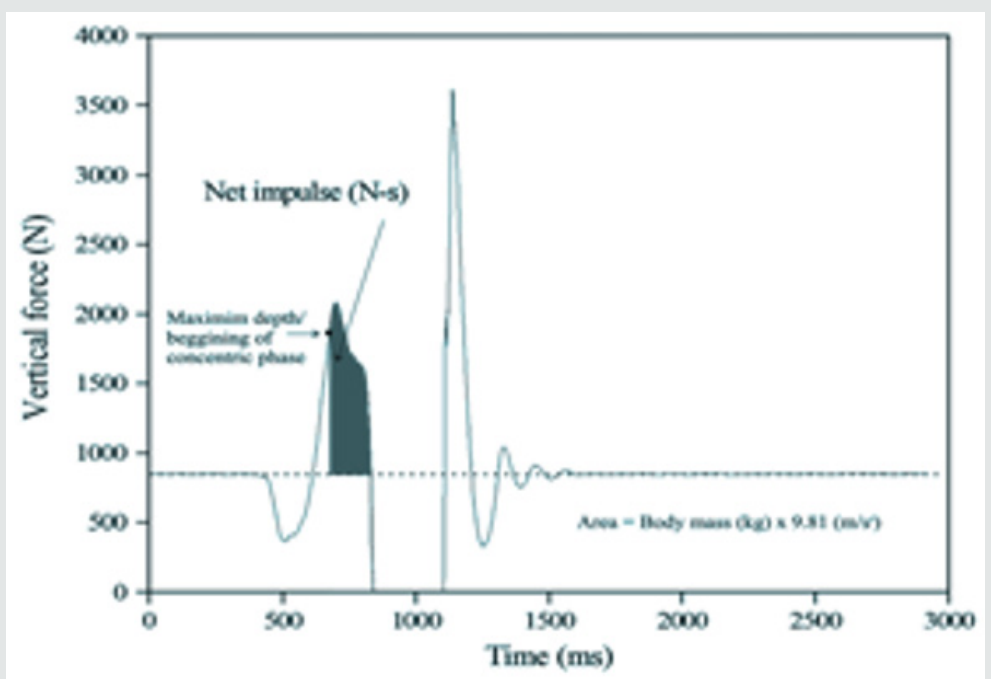

Figure 2: Force-time curve showing the determination of net vertical impulse.

\section{Judogi test procedures}

After performing a specific warm-up for upper limbs and familiarization by the athletes of two sets of two repetitions of grip holding on to a Judogi rolled around a bar, with 2 min intervals between them. Following $3 \mathrm{~min}$ of passive rest. The participants performed the JGST a study of [18] noted that hand-grip strength is a relevant indicator of predicting the results of competitions of young judokas, [19]. Which consisted of performing a maximum number of repetitions from elbow fully extended to elbow fully flexed, with the same grip position applied as that used during the familiarization exercises. A dynamic grip strength endurance seems to be a discriminating variable between judo athletes, probably because judo combat involves many elbow extensions and flexion in order to avoid the opponent's grip and to subdue them [20,21]. In the present study, the reliability was not tested in the baseline measures because it could provoke fatigue and interfere with the subsequent analysis.

\section{Rate of Perceived Exertion}

A rating of perceived exertion scales the study [22] was designed to show the general increase in perceived exertion, perception of aches or pain in the legs, translated into languages. Understood by the participating wrestlers, the participants were also asked to indicate, on an anatomical diagram of the anterior and posterior views of the body, the areas they perceived to experience most exertions during competitions. The participants were asked how much they perceived the exertion and which muscles or muscle groups they felt were involved $[23,24]$. Before the first comp and after each comp, the athletes were questioned about their perception of exertion RPE based on the 6-20 scale [25].

\section{Result}

Table 1 shows the mean and standard deviation values for strength parameters and perceptual Variables Before-comp and post-com (Comp.1, Comp.2, Comp.3 Comp.4 and comp.5). Analysis 
of variance showed that jump height $(\mathrm{F}=93.96, \mathrm{p}=0.001$, sig*= 0.003), and relative net vertical impulse obtained in the CMJ (F $=14.10, \mathrm{p}=0.001$, sig $=0.00)$ were affected by the competition. Besides, JGST $(\mathrm{F}=29.74, \mathrm{p}<0.001$, sig $=0.00)$ and RPE $(\mathrm{F}=35.66$, $\mathrm{p}<0.001$, sig $=0.00$ ) revealed significant differences over the competition. According to the post hoc analysis, the jump height decreased in post-comp two and three compared to the Beforecomp, as well as the power output decreased in post-comp two and three in comparison to Before-comp and post-comp1. The relative net vertical impulse showed a reduction in post-comp2 compared to Before-comp and post-comp1. There was a significant reduction of JGST performance in post-comp1, 2, 34, and 5 when compared to Before-comp. The RPE increased post- comp1, 2, 34, and 5 when compared to Before-comp and increased post-comp two and three when compared to post-comp one.

Table 1: Shows the mean and standard deviation values for strength parameters and perceptual Before-comp and post-com (Comp.1, Comp.2, Comp.3 Comp.4 and comp.5).

\begin{tabular}{|c|c|c|c|c|}
\hline & Vertical Jump height (cm) & $\begin{array}{l}\text { Impulsion of vertical } \\
\text { jump (Ns/kg) }\end{array}$ & Judo-Ji Test (rep) & RPE scale (6-20) \\
\hline Before.com (n=34) & $51.36 \pm 0.79$ & $4.97 \pm 0.42$ & $13,50 \pm 1,54$ & $10,76 \pm 1,98$ \\
\hline Post-Comp.1 (n=34) & $48.96 \pm 0.47^{*}$ & $4.46 \pm 0.26^{*}$ & $12,50 \pm 1,54^{*}$ & $12,58 \pm 1,67^{*}$ \\
\hline Post-Comp.2 (n=34) & $48.75 \pm 0.66^{*}$ & $4.87 \pm 0.40 *$ & $10,50 \pm 1,54^{*}$ & $13,47 \pm 1,82^{*}$ \\
\hline Post-Comp.3 (n=34) & 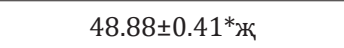 & $4.74 \pm 0.40 *$ ж & $11,58 \pm 1,54 *$ & $11,79 \pm 2,19 *$ \\
\hline Post-Comp.4 $(\mathrm{n}=34)$ & $48.73 \pm 0.87 *_{\dddot{2}}$ & $4.43 \pm 0.46 *$ ж & $12,44 \pm 1,86 *$ ж & $14,17 \pm 2,23 *$ ж \\
\hline Post-Comp.5 (n=34) & $47.91 \pm 0.73^{*}$ & $4.43 \pm 0.45 *$ & $11,08 \pm 1,70 *$ & $12,97 \pm 1,58^{*} \dddot{~}$ \\
\hline Sig & 0.00 & 0.00 & 0.00 & 0.00 \\
\hline
\end{tabular}

- Note: Data are expressed as means \pm standard deviation.

$\left.{ }^{*}\right)$ Significantly different from Before-Comp.

(ж) Significantly different from post-comp 1 ( $\mathrm{p}<0.05)$; Sig = significant

The RPE values of specific areas obtained after the judo competition are described in (Figure 3). The athletes reported RPE in ten areas after the simulated tournament, in which the forearms, Abdominal and quadriceps (42.07\%,27.08\% and,24 respectively) were the most frequently cited. It is important to highlight that the percentage of each area represents the number of individuals who marked it. When we compared the RPE between the upper

body (average scores of forearms, fingers, biceps, chest, trapezius and abdomen) and the lower body (average scores of quadriceps, abductors, anterior tibia and feet), no significant differences were found (RPE upper body: $14 \pm 5$, RPE lower-body: $16 \pm 3, p=0.001$, sig $=0.000)$. (Figure 3). Relative frequency of specific areas cited by the athletes and intensity of RPE values (Mean percentage \%) measured after a competition (Figure 4).

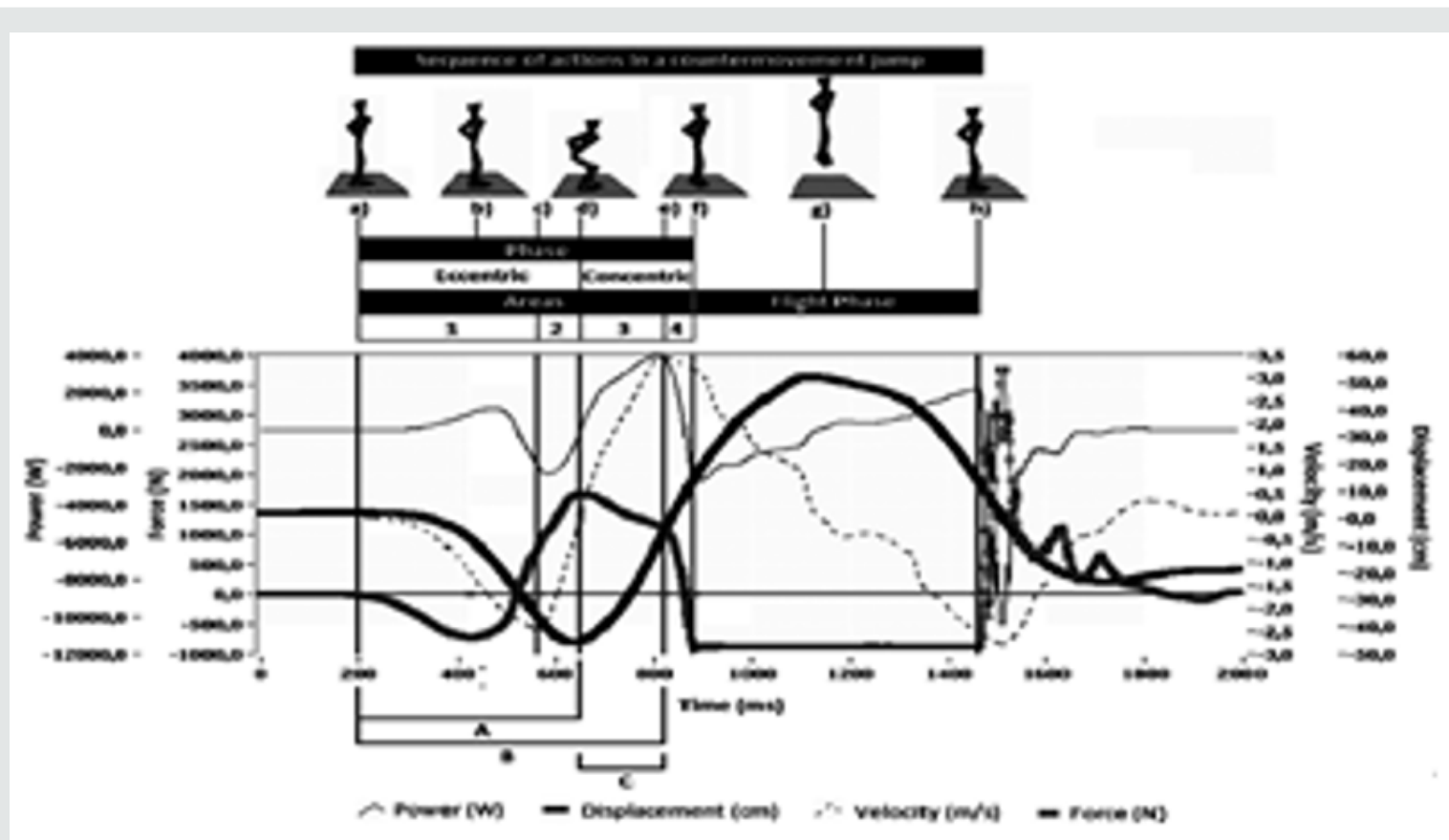

Figure 3: Dynamic and kinematic curves for the countermovement jump (sample output). 


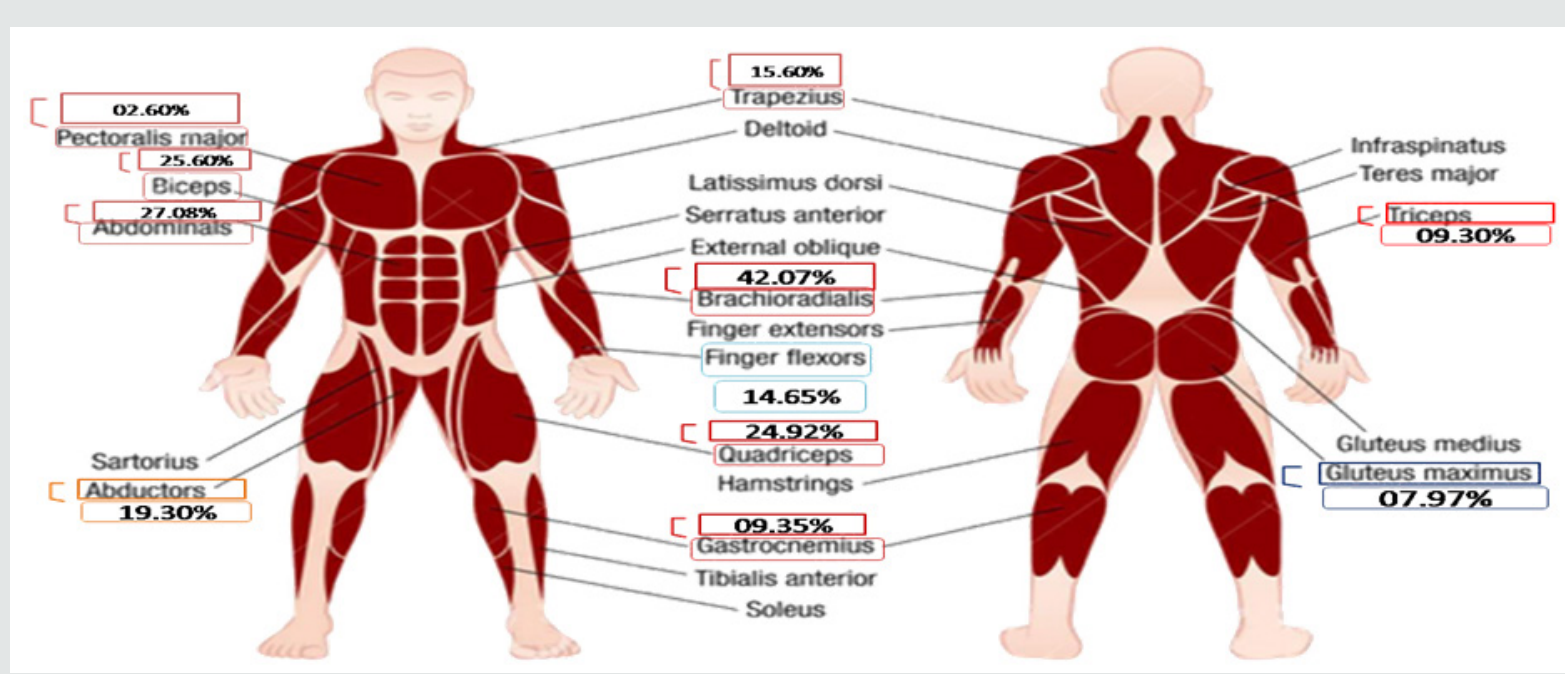

Figure 4: Relative frequency of specific areas cited by the athletes and intensity of RPE values (Mean percentage \%) measured after a competition.

\section{Discussion}

The initial hypothesis of [26] was confirmed concerning improvements in upper-body anaerobic power and capacity, lowerbody muscle power and strength endurance and body composition maintenance, but it was not confirmed concerning lower-body mechanical aerobic power maintenance [27]. Reviews identify these misapplications and propose solutions. Solutions include adoption of the term "intensity" in descriptions and categorizations of challenges imposed on an individual as they perform exercise. By using the RPE [28], followed by correct use of SI terms and units appropriate to the specific kind of exercise performed [29]. Such adoption must occur by authors and reviewers of sport and exercise research reports to satisfy the principles and practices of science and for the field to advance [30]. We found a significant decrease $(11.26 \%)$ of jump height obtained in the CMJ from the third competition and power output (decrease of $7.3 \%$ ) from the first competition. This normative data can be used in the design of training programs for female judoka, as well as evaluation after training, and in preparation for competition [31]. The results of $[32,33]$ study indicate that there was a higher alactic contribution compared with both aerobic and lactic contributions during the SJFT. which can be considered to be the most predominant system contributing to the actions (techniques) performed in the judo competitionThe purpose of this study was to analyze the real effects of competition on vertical jump performance, grip strength test and perceived effort responses. The hypothesis was accepted, as the competition induced a decrease of performance in both upper and lower limbs (tiredness effect) and provoked a progressive increase in the effort perception over the course of the competition. In the present study, the simulated Judo competition required to increase the external validity through the combat with different opponents and the correct competition time according to IJF rules (« International Judo Federation. (2018). New IJF Judo Refereeing Rules »).

\section{Study Limitation}

Two main limitations can be identified in our study: the shorter recovery period of the athletes between each match due to the post-assessments, in official judo competitions each athlete has at least three the time of the competition to recover. The absence of a control group submitted to a judo competition and the lack of control concerning the athlete's nutritional intake. The use of a control group in this study was difficult because athletes are normally submitted to some type of competition process. Additionally, some authors [34] consider that there would be "ethical problems with restricting a particular treatment to elite athletes". Although diet was not controlled in this study, athletes were not using any supplementation and were oriented to keep their normal nutritional habits throughout the study.

\section{Conclusion}

We conclude that the Algerian modality judo competitioninduced fatigue in both upper and lower limbs showed by the reduction in the number of repetitions in the JGST and the decrease in vertical jump performance (jump height and net vertical impulse) after first competitions. In addition, the overall effort perception increased progressively over the other consecutive competitions. Considering the effort perception in specific areas, a higher intensity was observed in forearms and quadriceps; however, in general, when comparing the upper and lower body, it seems that both underwent similar demands during a judo competition. From that, we recommend our judoka to combat with different opponents under the progression of time competition and adversary's ability for the success and rest of the competitions.

\section{References}

1. Andreato LV, Franchini E, de Moraes SMF, Pastório JJ, da Silva DF, et al. (2013) Physiological and Technical-tactical Analysis in Brazilian Jiu-jitsu Competition. Asian J Sports Med 4(2): 137-143. 
2. Andreato LV, Julio UF, Gonçalves Panissa VL, Del Conti Esteves JV, Hardt F, et al. (2015) Brazilian Jiu-Jitsu Simulated Competition Part II: Physical Performance, Time-Motion, Technical-Tactical Analyses, and Perceptual Responses. J Strength Cond Res 29(7): 2015-2025.

3. Belkadi A, Othman B, Mohamed SMBH, Gleyse J, Adel B, et al. (2015) Contribution to the Identification of the Professional Skills Profile of Coaches in the Algerian Sport Judo System. International Journal of Sports Science 5(4): 145-150.

4. Bonitch-Góngora JG, Bonitch-Domínguez JG, Padial P, Feriche B (2012) The effect of lactate concentration on the handgrip strength during judo bouts. Journal of Strength and Conditioning Research 26(7): 1863-1871.

5. Borg GA (1982) Psychophysical bases of perceived exertion. Med Sci Sports Exerc 14(5): 377-381.

6. Borg G, Ljunggren G, Ceci R (1985) The increase of perceived exertion, aches and pain in the legs, heart rate and blood lactate during exercise on a bicycle ergometer. Eur J Appl Physiol Occup Physiol 54(4): 343-349.

7. Brian Jones N, Ledford E (2012) Strength and Conditioning for Brazilian Jiu-jitsu 34(2): 60-69.

8. Buśko K, Pastuszak A, Kalka E (2017) Body composition and somatotype of judo athletes and untrained male students as a reference group for comparison in sport. Biomedical Human Kinetics 9(1): 7-13.

9. Detanico D, Dellagrana RA, Athayde MSdaS, Kons RL, Góes A (2017) Effect of a Brazilian Jiu-jitsu-simulated tournament on strength parameters and perceptual responses. Sports Biomech 16(1): 115-126.

10. Detanico D, Santos SGdos (2012) Especific evaluation in judo: a review of methods. Brazilian Journal of Kinanthropometry and Human Performance 14(6): 738-748.

11. Enoka RM, Stuart DG (1992) Neurobiology of muscle fatigue. J Appl Physiol (1985) 72 (5): 1631-1648.

12. Eston R (2012) Use of ratings of perceived exertion in sports. Int J Sports Physiol Perform 7(2): 175-182.

13. Eston RG, Lambrick DM, Rowlands AV (2009) The perceptual response to exercise of progressively increasing intensity in children aged 7-8 years: Validation of a pictorial curvilinear ratings of perceived exertion scale. Psychophysiology 46(4): 843-851.

14. Foster C, Florhaug JA, Franklin J, Gottschall L, Hrovatin LA, et al. (2001) A new approach to monitoring exercise training. J Strength Cond Res 15(1): 109-115.

15. Franchini E, Branco BM, Agostinho MF, Calmet M, Candau R (2015) Influence of linear and undulating strength periodization on physical fitness, physiological, and performance responses to simulated judo matches. J Strength Cond Res 29(2): 358-367.

16. Franchini E, Del Vecchio FB, Ferreira Julio U, Matheus L, Candau R (2015) Specificity of performance adaptations to a periodized judo training program. Revista Andaluza de Medicina del Deporte 8(2): 67-72.

17. Franchini E, Del Vecchio FB, Matsushigue KA, Artioli GG (2011) Physiological profiles of elite judo athletes. Sports Med 41(2): 147-166.

18. Franchini E, Miarka B, Matheus L, Vecchio FD (2011) Endurance in judogi grip strength tests: comparison between elite and non-elite judo players. Archives of Budo pp. 7(1).
19. Franchini E, Sterkowicz S, Szmatlan-Gabrys U, Gabrys T, Garnys M (2011) Energy system contributions to the special judo fitness test. Int J Sports Physiol Perform 6(3): 334-343.

20. Gheller RG, Dal Pupo J, Ache-Dias J, Detanico D, Padulo J, et al. (2015) Effect of different knee starting angles on intersegmental coordination and performance in vertical jumps. Hum Mov Sci 42: 71-80.

21. Gutierrez-Sanchez A, Soria Dominguez A, Pérez-Turpin J, Cortell-Tormo J, Suarez Llorca C (2011) Importance of hand-grip strength as an indicator for predicting the results of competitions of young judokas 7: 167-172.

22. Houcine A, Ahmed A, Saddek Z (2014) Designing a Software to Count the Body Composition and Somatotype and its Role in Pursing the Morphological State of Spotsmen. AASRI Procedia 8: 38-43.

23. International Judo Federation (2018) New IJF Judo Refereeing Rules (s. d.) Consulté 28 janvier 2018, à l'adresse.

24. Kirby TJ, McBride JM, Haines TL, Dayne AM (2011) Relative net vertical impulse determines jumping performance. J Appl Biomech 27(3): 207-214.

25. Lambert MI, Borresen J (2010) Measuring training load in sports. Int J Sports Physiol Perform 5(3): 406-411.

26. McGarry T, O’Donoghue P, Sampaio AJde E, Sampaio J (2013) Routledge Handbook of Sports Performance Analysis. Routledge pp.512.

27. Monteiro L, Massuça L, Garcia Garcia JM, Carratala V, Proença J (2011) Plyometric muscular action tests in judo-and non-judo athletes 19(4): 287-293.

28. Nilsson J, Csergö S, Gullstrand L, Tveit P, Refsnes PE (2002) Work-time profile, blood lactate concentration and rating of perceived exertion in the 1998 Greco-Roman Wrestling World Championship. J Sports Sci 20(11): 939-945.

29. Papacosta E (2015) Training, taper and recovery strategies for effective competition performance in judo (Thesis) pp.

30. Pithon MM (2013) Importance of the control group in scientific research. Dental Press J Orthod 18(6): 13-14.

31. Slimani M, Davis P, Franchini E, Moalla W (2017) Rating of Perceived Exertion for Quantification of Training and Combat Loads During Combat Sport-Specific Activities: A Short Review. J Strength Cond Res 31(10): 2889-2902.

32. Sterkowicz-Przybycien KL, Fukuda DH (2014) Establishing normative data for the special judo fitness test in female athletes using systematic review and meta-analysis. J Strength Cond Res 28(12): 3585-3593.

33. Weaving D, Jones B, Till K, Marshall P, Earle K, et al. (2017) Quantifying The External And Internal Loads Of Professional Rugby League Training Modes: Consideration For Concurrent Field-Based Training Prescription. J Strength Cond Res pp.

34. Winter EM, Abt G, Brookes FBC, Challis JH, Fowler NE, et al. (2016) Misuse of "Power" and Other Mechanical Terms in Sport and Exercise Science Research. J Strength Cond Res. 30(1): 292-300 


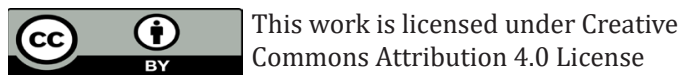

To Submit Your Article Click Here:

Submit Article

$\begin{gathered}\text { Orthopedics and Sports Medicine } \\ \text { Open Access Journal } \\ \text { Assets of Publishing with us } \\ \text { - Global archiving of articles } \\ \text { - Immediate, unrestricted online access } \\ \text { - Rigorous Peer Review Process } \\ \text { - Authors Retain Copyrights } \\ \text { - Unique DOI for all articles }\end{gathered}$

